\title{
Informationen für die Zukunftsplanung
}

Ulrike Pech und Hubert Oppl

Während anfänglich die Informationsversorgung des Managements im Vordergrund stand, versteht sich Controlling heute als Bestandteil eines umfassenden Managementinformationssystems, das Steuerungsgrößen für die Unternehmensplanung sowie die Kontrolle des Grades der Zielerreichung zur Verfügung stellt. Dabei geht es nicht nur um monetäre Steuerungsaufgaben, vielmehr geht es - je nach unternehmerischen Zielsetzungen - auch um »weiche "Unternehmensbelange.

Controlling kommt heute - neben dem unverzichtbaren Aufgabenbereich der monetären Unternehmenssteuerung auch eine Beratungsfunktion des Managements in der strategischen Unternehmensausrichtung zu: regionale und angebotsbezogene Domänenzentrierung, Marktstärke versus Marktgröße, ideelle Positionierung $\mathrm{u}$. a. Somit hat sich Controlling mittlerweile weit über die Diagnose situativer Soll-Ist-Abweichungen hinaus zu einem Instrument der prospektiven strategischen Unternehmenssteuerung entwickelt.

Im Sozialbereich sieht sich ein Controlling den Besonderheiten der Leistungserstellung gegenüber, denn der soziale Dienstleistungssektor grenzt sich in mehrfacher Hinsicht von anderen Wirtschaftszweigen ab. In aller Regel werden dort sogenannte meritorische Güter »produziert«. Sie können zwar privatwirtschaftlich angeboten werden, deren ausschließliche marktwirtschaftliche Steuerung wird politisch jedoch nicht akzeptiert (z. B. Gesundheitsversorgung) oder kann nicht akzeptiert werden (z. B. Rettungswesen). Das führt vielfach dazu, dass die jeweiligen Güter zu überhöhten Kosten produziert oder dass die Wünsche der Nachfrager und Kunden verfehlt werden. Weiterhin sind die angebotenen Güter überwiegend nicht lagerfähig, sie können nur durch die Mitwirkung eines »externen Faktors «, nämlich dem Kunden, erstellt werden. Schließlich fehlen die Möglichkeiten einer Marktsteuerung; zumindest sind sie erheblich eingeschränkt.

Wenngleich die Umsätze in der Sozialwirtschaft zu rund 80 Prozent über »Preise für Leistungen « finanziert werden, rührt die fehlende Marktsteuerung daher, dass die Leistungsentgelte weitgehend aus öffentlichen oder parastaatlichen Kassen stammen. Über eine Vielzahl von Wegen (Planung) und Instrumenten (Kostenfestsetzungen) greifen die öffentlichen Hände in das »Marktgeschehen« ein und machen es zum Teil unkalkulierbar.

Ein weiterer Einflussfaktor besteht in den vitalen Interessen einer großen Gruppe von Produzenten sozialer Dienst-

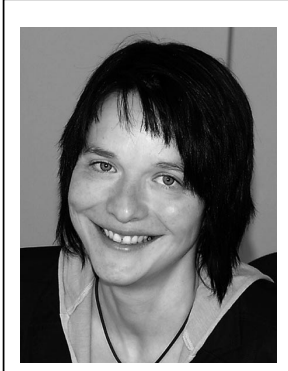

Prof. Dr. Ulrike Pech ist Hochschullehrerin im Wirtschaftsbereich an der Staatlichen Studienakademie Thüringen, Berufsakademie Gera. Sie vertritt die Fächer Rechnungswesen (Buchführung, Kosten- und Leistungsrechnung), Bilanzierung, Steuern und Controlling und ist Studienrichtungsleiterin Handel. Sie studierte Betriebswirtschaftslehre und Wirtschaftspädagogik an der Friedrich-Schiller-Universität Jena und arbeitete dann als Abteilungsleiterin Wirtschaft bei einer gemeinnützigen Trägergesellschaft mit 700 Mitarbeitern. E-Mail ulrike.pech@ba-gera.de

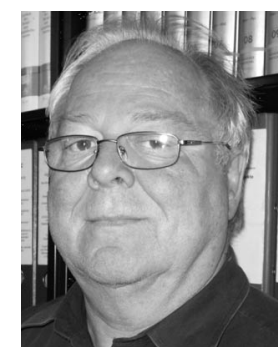

Prof. Dr. Hubert Oppl ist Diplom-Sozialpädagoge und war bis 1997 an der Katholischen Stiftungsfachhochschule München Hochschullehrer für Sozialarbeit, Pädagogik und Unternehmensführung in der Sozialwirtschaft. Managementerfahrungen bei sozialwirtschaftlichen Unternehmen sammelte er dann bei den Franziskanerinnen von Reute, Oberschwabenkliniken sowie der Refugium AG. Danach machte er sich mit mehreren Unternehmen selbstständig. Schwerpunkte seiner Beratungen für die Sozialwirtschaft sind Finanzierungslösungen für Sozialimmobilien sowie Lösungen der betrieblichen Altersvorsorge in der Sozialwirtschaft.

E-Mail hubert.oppl@carefuture.de

leistungen, erfolgreich substanzielle ideelle Ziele zu erreichen sowie besondere Ressourcen (ehrenamtliche Mitarbeit, Spenden) zu erschließen und an sich binden zu können.

Nach wie vor ist die Sozialwirtschaft von der kleinräumigen Unternehmensstruktur der Gründerzeit der Wohlfahrtsverbände geprägt. Allerdings entstehen zunehmend bei allen Anbietergruppen (gewerblich, öffentlich sowie freigemeinnützig) größere Unternehmenszusammenschlüsse. 
Dieser Trend aber auch die wachsende Komplexität des sozialen Dienstleistungssektors schlechthin konfrontieren ein Controlling mit nachhaltigen multiplen Aufgaben: Das Controlling muss eine zukunftsfeste Balance zwischen Wettbewerbsstrategie und Sozialwohlstrategie (Miegel/Ottnad), zwischen Wirtschaftlichkeit und Menschlichkeit (Sr. Basina Kloos) entwickeln.

\section{Wie Sozialwirtschaft funktioniert}

Die Grundsätze des Wirtschaftens in der Sozialarbeit unterscheiden sich nicht wesentlich von denen anderer Wirtschaftsbereiche. Ob eine spezielle sozialwirtschaftlich orientierte Betriebswirtschaftslehre - analog dem Banken-, Handels-, Industrie oder Dienstleistungssektor - sinnvoll oder auch erforderlich sein ist, kann hier offen bleiben. Die Anwendbarkeit bewährter betrieblicher Steuerungs- sowie Planungsinstrumente steht außer Frage. Daher können nachfolgend Möglichkeiten und Anwendungsbereiche eines operativen sowie strategischen Controllings vertieft werden.

Die multiplen Einflussfaktoren auf Managemententscheidungen in der Sozialwirtschaft leiten sich nicht allein aus betrieblichen, marktorientierten oder weiteren engeren Faktoren des unternehmerischen Umfeldes ab. Verschärfend kommt hinzu, dass gerade bei den Hauptumsatzträgern in der Sozialwirtschaft (Gesundheitsdienstleistungen, Pflege, Dienstleistungen für behinderte Menschen) kontinuierlich staatliche »Übergriffe» drohen, die ein vorausschauendes unternehmerisches Handeln erschweren. Daher steht im Mittelpunkt gegenwärtiger und künftiger unternehmerischer Positionierungen die Frage: Wie kann Zukunftssicherung durch Marktorientierung und aktive Marktgestaltung gelingen. Entscheidend ist hierfür ein bewährtes Instrumentarium an Früherkennungsparametern, die die unternehmerische Flexibilität erhöhen. Für eine wirksame, erfolgreiche und nachhaltige Steuerung treten hierbei Vergangenheitsdaten zunehmend in den Hintergrund.

\section{Bedeutsame Controllingfunktionen sind}

- Führungsunterstützung: Servicefunktionen und Dienstleistungen, um der Unternehmensführung zu ermöglichen, ihren Managementaufgaben nachzukommen (Planen, Entscheiden, Koordinieren, Kontrollieren)
- Informationsfunktion: systematische Informationen, in der richtigen Qualität und Menge, zum richtigen Zeitpunkt, beim richtigen Empfänger

- Koordinationsfunktion: Abstimmung aller mit finanziellen Ressourcen behafteten Aktivitäten und Koordination der erforderlichen Informationen

- Sicherung der Unternehmenszukunft: Initiierung und Koordinierung von Innovationsprozessen, um Flexibilität und dauerhaften Unternehmenserfolg zu ermöglichen

Entsprechend den jeweiligen Akzenten wird zwischen strategischem Controlling (die richtigen Dinge tun) sowie operativem Controlling (die Dinge richtig tun) unterschieden (vgl. untenstehende Tabelle).

Das für die Unternehmen der Sozialwirtschaft vielfach verwendete Synonym der »Non-Profit-Organisationen « ist unzutreffend und irreführend zugleich. Es vermischt in unzulässiger Weise für den Teilbereich der (frei-) gemeinnützigen Wohlfahrtspflege die Trägerebene (in der Regel eingetragener Verein oder Körperschaft) und die Ebene ihrer wirtschaftlichen Geschäftsbetriebe (Zweckbetriebe). Daher hat sich in der Literatur die Trennung zwischen Organisationsebene sowie Unternehmensebene eingebürgert. Indessen schärft diese Trennung die Analysemöglichkeiten nur unwesentlich. Die vielfach gegenüber der gewerblichen Wirtschaft benutzte Trennung zwischen Gewinnerzielungspriorität sowie Priorität der Sinnstiftung, Wertverwirklichung sowie Bedarfsdeckung führt zu einer erschwerten oder sogar unmöglichen Zielintegration. Die Verwirklichung der letztgenannten Ziele hängt entscheidend von den verfügbaren wirtschaftlichen Mitteln ab. Sie kommen entweder aus Eigenmitteln, aus ehrenamtlichem Engagement, aus öffentlichen oder parastaatlichen Kassen oder aber aus Unternehmensgewinnen der eigenen Zweckbetriebe.

Gegenüber den seit langem rückläufigen »Mitteln eigener Art « sowie der Transferzahlungen aus öffentlichen Töpfen gewinnt wirtschaftlich erfolgreiches Unternehmertum zunehmend an Bedeutung. Daher sind die wirtschaftlichen Geschäftsbetriebe gemeinnütziger Vereine oder auch die gemeinnützigen Kapitalgesellschaften (als immer häufiger gewählte Rechtsform für ehemalige Zweckbetriebe) wie jedes andere Wirtschaftsunternehmen auch darauf angewiesen, Gewinne zu erzielen, um daraus nicht gewinnfähige Handlungsbereiche (z. B. Beratung) finanzieren zu können. Eine darauf bezogene Erfolgsorientierung steht auch nicht

\begin{tabular}{|l|l|l|}
\hline & operatives Controlling & strategisches Controlling \\
\hline Ziele & $\begin{array}{l}\text { Operationalisieren der (strategischen) Ziele, } \\
\text { Bilanz, Erfolgs-, Finanz- und Liquiditätsplanung; } \\
\text { Abweichungsanalyse } \\
\text { alle Managementebenen; } \\
\text { sämtliche marktab- bzw. zugewandten Bereiche }\end{array}$ & $\begin{array}{l}\text { Aufzeigen, Sicherung und Ausbau von } \\
\text { Erfolgspotentialen } \\
\text { durch Antizipation von Änderungen der } \\
\text { Rahmenbedingungen } \\
\text { oberste Managementebene, } \\
\text { bereichsübergreifend - ganzheitlich } \\
\text { gering strukturierte Problemstellungen, } \\
\text { hohe Komplexität } \\
\text { Strategische Planungs- und } \\
\text { Kontrollsysteme }\end{array}$ \\
Instrumente & $\begin{array}{l}\text { klar strukturiert, geringe Unsicherheit } \\
\text { Zeitbezug }\end{array}$ & $\begin{array}{l}\text { Operative Planungs- und Kontrollsysteme } \\
\text { kurzfristig, bis zu 5 Jahren }\end{array}$ \\
\hline
\end{tabular}


im Widerspruch zu den Gemeinnützigkeitsprivilegien der Abgabenordnung. Erzielte Gewinne müssen lediglich im Gemeinnützigkeitskreislauf verbleiben.

Schließlich hat die Abwendung vom retrospektiven Kostenersatz der Vergangenheit zur Vereinbarung prospektiver Preise in vielen Leistungsbereichen neben weiteren Einflussfaktoren einer wachsenden Marktregulierung den Zwang zur Wirtschaftlichkeit beträchtlich erhöht. Nachfrageschwankungen sowie damit einhergehende Kostenrisiken sind nur begrenzt einpreisbar, sie müssen über intelligente Steuerungsinstrumente abgefedert werden. Gerade wegen der nur eingeschränkten Einflussnahme auf nach wie vor administrierte Preise kommt dem zeitnahen Informationsfluss - beispielsweise bei Belegungsschwankungen in vollstationären Pflegeeinrichtungen, Krankenhäusern oder Reha-Kliniken - sowie der darauf aufbauenden Gegensteuerungsmöglichkeiten - beispielsweise durch Steuerung des Personaleinsatzes - eine besondere Bedeutung zu. Sachkundiges Controlling wird daher in vielen sozialwirtschaftlichen Unternehmen zur Überlebensfrage.

\section{Controlling, Qualitätssicherung und Qualitätsmanagement}

Gemeinwirtschaftliche Unternehmen sowie ihre Gesellschafter oder Träger stehen in einer besonderen Verpflichtung, einen Beitrag zur Humanisierung der Gesellschaft zu leisten. Sie verstehen sich insoweit als Bestandteil des »sozialen Immunsystems«. Soziale Schäden entstehen dann oder können nicht behoben werden, wenn sie in ihrer sinnstiftenden sowie wertverwirklichenden Effektivität nachlassen (vgl. Mayer, S. 691 ff.).

Die Sozialwirtschaft steht vor einer doppelten Modernisierungskrise: zum einen führt ein zunehmender Verlust an Systemvertrauen bei Bürgern zu einem wachsenden Misstrauen dem »Hilfebürokratismus «; zum anderen stellt die wachsende Ökonomisierung der Sozialwirtschaft

\section{Literatur}

Botta, V. (Hg.): Controlling in Theorie und Praxis, Aachen 1999.

Fischer, R.: Dienstleistungs-Controlling. Grundlagen und Anwendungen, Wiesbaden 2000.

Hauser, A.: Sozial-Management. Praxishandbuch soziale Dienstleistungen, Neuwied 2000.

Mayer, E.: Controlling in Nonprofit- Organisationen und -Unternehmen, in: Gablers Wirtschaftslexikon, S. 691 ff., 13. Auflage Wiesbaden 1993.

Oppl, H./Pech, U.: Fit für den Markt, in Sozialwirtschaft, Zeitschrift für Sozialmanagement, 2/2009, S. 35 ff.

Ottnad/A./Wahl, St./Miegel, M: Zwischen Markt und Mildtätigkeit, Die Bedeutung der Freien Wohlfahrtspflege für Gesellschaft, Wirtschaft und Beschäftigung, München 2000.

Pech, U.: Bildungscontrolling, Aachen 2001.

Strunk, A.: Dienstleistungscontrolling. Strategien zur Innovationssteuerung im Sozial- und Gesundheitssystem, Baden-Baden 1996.

Schubert, H.: Sozialmanagement: zwischen Wirtschaftlichkeit und fachlichen Zielen, Wiesbaden 2005.

Weber, J: Controlling, in Handelsblatt Wirtschafts-Lexikon, Band 3, S. 1125 ff. Stuttgart 2006. diese vor eine Zerreißprobe. Stehen sich Ökonomie und Menschlichkeit unvereinbar gegenüber oder kann der fachgerechte Einsatz von Instrumenten der Betriebswirtschaft zu einer Optimierung personenbezogener Dienste führen, ja führt mehr Ökonomie zu mehr Menschlichkeit, wie es Sr. Basina Kloos von den Waldbreitbacher Franziskanerinnen nahelegt?

Dass sich ein Controlling aber auch inhaltlichen Fragestellungen zuwenden muss, ist der Tatsache geschuldet, dass Sozialleistungen immer Dienstleistungen sind, welche durch die Merkmale der Nichtlagerfähigkeit, des Uno-actu-Prinzips und des Einbezugs eines externen Faktors gekennzeichnet sind. Allerdings sind die häufig verwendeten klassischen Effizienzberechnungen im Dienstleistungsbereich inhaltlich leer. Es könnten zwar beispielsweise die Kosten pro behandeltem Patient berechnet und aus Effizienzgründen versucht werden, dieses Verhältnis zu verbessern. Doch was nützt diese Verbesserung ohne die Frage nach der Qualität? Folglich müssen inhaltliche Fragestellungen nach der Qualität der Leistungserbringung in die Betrachtung durch ein sach- und zielgerechtes Controlling mit einbezogen werden.

Die Literatur lässt keinen Zweifel daran: Controlling ist ein unverzichtbares Instrument erfolgreicher betrieblicher Steuerung, weil rationelle sowie effektive Mittelsteuerung ein substanzielles Instrument der Überlebenssicherung darstellt. Die Modellierung der Instrumente sowie ihr zielorientierter Einsatz differenzieren sich danach aus, ob es sich um »bösartige« oder »zahme« soziale Aufgabenstellungen handelt. Während sich zahme soziale Probleme (Ablauf von Pflegeereignissen) einer regulären sowie kalkulierbaren wirtschaftlichen Darstellung öffnen, sind bösartige (z. B. Kostendämpfungsaufgaben, Effizienzverbesserung) in aller Regel von Wertkonflikten begleitet, die besondere konfliktgeeignete Strategien erfordern (ebenda S. 694). Entsprechende strategische Orientierungen stellen völlig andere Anforderungen an das Management und an seine Handlungsgrundlagen. Geeignete Zielkompetenz für zukunftsorientiertes Denken und Handeln erfordert hinreichend belastbare operative und strategische Werkzeuge, die realitätsbezogene Ziele und Aufgaben zwischen Wünschbarem und Machbarem definieren. Diese Werkzeuge leiten sich aus einem Werkzeugkasten ab, der aus der Vernetzung operativer sowie strategischer Regelkreise gebildet werden muss (ebenda S. 701).

\section{Resümee}

Operatives Controlling sowie Marketing und Qualitätsmanagement erlauben eine aktive Gewinnsteuerung sowie die Beseitigung operativer Erfolgsengpässe, strategisches Controlling sowie Marketing und Qualitätsmanagement sichern durch eine antizipierende Analyse sowie Modellrechnungen der Änderungen von Rahmenbedingungen und ihrer Folgen die nachhaltige Existenzsicherung des Unternehmens auch jenseits des (operativen) Zeithorizonts.

Insoweit ist Controlling eine kontinuierliche Verpflichtung zur Unternehmenssteuerung, damit gemeinwirtschaftliche Unternehmen - gestützt auf Erfolge im regulären Geschäftsbetrieb - ihre soziale Immunitätswirkung auch künftig entfalten können. 